\title{
Comparison of Diffusion and Perfusion Techniques in Differentiating High Versus Low Grade Pediatric Brain Tumors
}

Eric Chen ${ }^{1}$, Chang Ho², Benjamin Gray², Jason Parker², Emily Diller², Qiuting Wen², Yu-Chien $\mathrm{Wu}^{2}$, Gregory Anthony ${ }^{2}$, Paul R. Territo ${ }^{2}$

${ }^{1}$ Indiana University School of Medicine, ${ }^{2}$ Indiana University School of Medicine Department of Radiology and Imaging Sciences

Background/Objective: Brain tumors are the most common solid cancer in children and cause significant mortality and morbidity. We compare the effectiveness of different parameters in predicting tumor grade between dynamic contrast enhancement (DCE), intravoxel incoherent motion (IVIM), dynamic susceptibility contrast (DSC) perfusion and diffusion weighted imaging (DWI).

Methods: A retrospective blinded review of pediatric brain tumors with DCE, IVIM, DWI, and DSC was performed. Parametric maps were registered to T2 weighted images. Volumetric regions of interest (ROI) were manually segmented from solid tumor components for each patient by a neuroradiologist $(\mathrm{CH})$, neuroradiology fellow (BG), and medical student (EC). Resulting mean values for parameters from DCE (Ktrans, Kep, Ve, Vp,), IVIM (D, D*, f), DSC (rCBV) and DWI (ADC) were compared using Student's t-test for high- and low-grade tumor groups based on WHO grading from pathology. For significant parameters, receiver operating characteristic (ROC) analysis with area under curve (AUC) was performed.

Results: 20 subjects were included with 9 low grade and 11 high grade tumors. Significant differences between low versus high grade were demonstrated for $D(10-3 \mathrm{~mm} 2 / \mathrm{s})(1.4 \pm 0.4 \mathrm{vs}$ $0.9 \pm 0.2, p=0.01), f(0.04 \pm 0.02$ vs $0.07 \pm 0.02, p=0.02), A D C(10-3 \mathrm{~mm} 2 / \mathrm{s})(1.4 \pm 0.4$ vs $0.9 \pm 0.3$, $p=0.009)$ and rCBV $(2.2 \pm 0.9$ vs $4.7 \pm 2.1, p=0.003)$. No significant difference was found for $D^{*}$ or any DCE parameter. AUC from ROC was similar for all significant parameters [D $(0.81$, $p=0.003) ; f(0.80, p=0.003) ; \operatorname{ADC}(0.83, p=0.001)$; rCBV $(0.83, p=0.0005)]$.

Conclusion: $\mathrm{D}$ and $\mathrm{f}$ parameters from IVIM can significantly differentiate high versus low grade pediatric brain tumors similar to ADC and rCBV. Conversely, no DCE parameter was significant.

Scientific Implications: The results will assist the selection of MRI sequences that best predict tumor grade, as well as guide tumor biopsy for the most aggressive tumor portions. Further study of these techniques may correlate with molecular profiling and predict outcome. 